\title{
Clinical Evaluation of the NS Endo-Inserter, a Novel Donor Inserter for Descemet's Stripping Automated Endothelial Keratoplasty
}

\author{
Akira Kobayashi Hideaki Yokogawa Natsuko Mori Tsubasa Nishino \\ Kazuhisa Sugiyama \\ Department of Ophthalmology, Kanazawa University Graduate School of Medical Science, \\ Kanazawa, Japan
}

\section{Keywords}

NS Endo-Inserter · Descemet's stripping automated endothelial keratoplasty · Bullous keratopathy · Argon laser iridotomy

\begin{abstract}
Purpose: This study investigated the clinical outcomes achieved with a newly developed donor inserter (NS Endo-Inserter [NSI], HOYA Co., Ltd., Tokyo, Japan) for Descemet's stripping automated endothelial keratoplasty (DSAEK) in Japanese eyes with bullous keratopathy secondary to argon laser iridotomy (BK-ALI). The NSI device utilizes pressure flow to push the DSAEK donor tissue into the anterior chamber. Methods: Six eyes of 6 patients $(1$ male, 5 females; mean age, 78.5 years) with BK-ALI were enrolled. Donor tissue was pushed into the anterior chamber using the NSI. Intraoperative complications, graft dislocation, and iatrogenic primary graft failure were recorded for all eyes. Six-month postoperative central donor endothelial cell densities $(E C D)$ were measured prospectively and compared with preoperative values, along with 6-month best corrected visual acuity (BCVA). Results: In all cases, donor loading onto the NSI spatula and donor insertion into the anterior chamber using the NSI was smooth and successful; no intraoperative complications were noted. There were no cases of graft dislocation or IPGF. Postoperative ECD was 2,187.2 cells $/ \mathrm{mm}^{2}$ (mean loss, $14.8 \%$ ) and mean BCVA increased from 0.27 decimal to 0.8 at 6 months. Conclusion: In this small preliminary case
\end{abstract}


series, clinical outcomes for patients with BK-ALI undergoing DSAEK using the NSI were comparable or better than those achieved with conventional DSAEK insertion techniques.

(C) 2019 The Author(s)

Published by S. Karger AG, Basel

\section{Introduction}

Descemet's stripping automated endothelial keratoplasty (DSAEK) is now widely performed for treatment of endothelial dysfunction [1]. The advantages of DSAEK over standard penetrating keratoplasty are as follows: complete elimination of surface corneal incisions or sutures, preservation of much of the structural integrity of the cornea, minimal refractive changes, and less rejection [2]. However, endothelial cells of the DSAEK grafts may sustain greater early endothelial cell loss due to the increased manipulation compared to penetrating keratoplasty [2]. In all DSAEK procedures, donor insertion into the anterior chamber is thought to be critical in regard to surgical trauma to the donor endothelial cells [3]. During the early development of DSAEK procedures, donor insertion techniques focused on tissue folding and insertion with forceps [1-3]. The forceps donor insertion technique is difficult to perform in Asian eyes because they tend to have shallow anterior chambers with small corneal diameters and thus, it is nearly impossible to avoid severe endothelial cell damage during insertion [4]. Subsequent development of this procedure included a donor pull-through technique using an intraocular lens sheet glide [4], Busin glide [5], or the double-glide technique (intraocular lens sheet glide-assisted Busin glide technique) [6]. Initial studies with these newer pullthrough techniques suggested reduced endothelial cell loss [4-6] compared with results from large case series using the conventional taco-fold forceps techniques. Following these advancements, Professor Donald Tan from Singapore developed a new disposable DSAEK glide (Tan EndoGlide, AngioTech, Reading, PA/Network Medical Products, North Yorkshire, UK) to optimize the pull-through technique [7]. Subsequently, Dr. William Neusidl developed a new disposable DSAEK inserter named the Neusidl Corneal Inserter (NCI; Fischer Surgical, Imperial, MO, USA) to further minimize endothelial cell damage [8].

The purpose of the present study was to investigate the preliminary clinical outcomes achieved with a newly developed donor inserter (NS Endo-Inserter [NSI], Hoya Co., Ltd., Tokyo, Japan) created by Professor Kohji Nishida and Dr. Takeshi Soma from Osaka University $[9,10]$ for DSAEK in Japanese eyes with bullous keratopathy secondary to argon laser iridotomy (BK-ALI). Since the causative diseases of bullous keratopathy in Asian countries are quite different to those of Western countries [11], only BK-ALI patients were selected in this first preliminary study to simplify the interpretation of results.

\section{Materials and Methods}

\section{Patients and Methods}

Six eyes of 6 patients ( 1 male, 5 females; mean age, 78.5 years) with BK-ALI were enrolled. All DSAEK surgeries were performed by 2 expert surgeons (A.K. and H.Y.) at the Department of Ophthalmology, Kanazawa University Hospital. All patients read and signed an informed consent document prior to enrollment. Donor tissue was pushed into the anterior chamber using the NSI. Intraoperative complications, graft dislocation, and iatrogenic primary graft failure (IPGF) were recorded for all eyes. Six-month postoperative central donor endothelial cell densities (ECD) using a Noncon Robo microscope (Konan Medical Inc., Hyogo, Japan) and 
the center method as outlined by the manufacturer's software were measured and compared with preoperative values, along with 6-month best corrected visual acuity.

\section{Surgical Technique Using the NS Endo-Inserter}

All DSAEK procedures were performed as previously described [6] except for the donor insertion step. In brief, all precut DSAEK donors were internationally shipped from a US eye bank (CorneaGen ${ }^{\mathrm{TM}}$, Seattle, WA, USA). Donor tissue was transferred to a punching system and cut with a 7.5- or 8.0-mm diameter punch (Barron Donor Cornea Punch, Katena Products Inc., Denville, NJ, USA). For patients with cataracts and endothelial failure (Cases 1, 2, 5, and 6), a phacoemulsification procedure was performed from a 2.4-mm clear corneal temporal incision just prior to DSAEK. For one patient with aphakia (Case 3), intraocular lens scleral fixation was simultaneously performed just prior to DSAEK. Three corneal fenestrations were created to drain the interface fluid. Then, a small inferior iridectomy at the 6 o'clock position was created using a 25-gauge vitreous cutter (MID Labs, San Leandro, CA, USA) under continuous irrigation from a 25-gauge anterior chamber maintainer (25-gauge DSAEK Chamber Maintainer, catalog \# AE-7802, ASICO, Westmont, IL, USA) to prevent papillary air block after surgery. The detailed specifications of the NSI have been described elsewhere [9]. In brief, the NSI consists of a $2.5-\mathrm{mL}$ syringe and a main body with a flexible platform at its front end. Before loading the donor onto the NSI, the syringe was filled with balanced salt solution (BSS; Fig. 1a). The DSAEK graft was then placed onto the platform with the endothelial cell surface facing upward (Fig. 1b). An appropriate ophthalmic viscoelastic device (OVD; Viscoat, Alcon, Fort Worth, TX, USA) was applied to the endothelial surface of the graft, and the flexible platform with the graft was gently pulled into the cartridge resulting in coiling of the donor (Fig. 1c). The graft and BSS were then delivered into the anterior chamber by gentle depression of the syringe plunger through a premade $4.6-\mathrm{mm}$ clear corneal incision using a specialized DSAEK knife (catalog \# DSK-46, KAI Industries, Gifu, Japan); this incision is designed to form a tight seal (Fig. 1d). Before insertion of the NSI, the anterior chamber maintainer was turned off. During corneal graft delivery, the closed system prevents anterior chamber collapse (Fig. 1e). After securing the wound with an interrupted 10-0 nylon suture, air was injected into the anterior chamber to press the donor tissue against the recipient cornea (Fig. 1f). Corneal massage was performed in all cases to eliminate residual fluid at the recipient-donor interface. The anterior chamber was kept full of air and the patient was instructed to lie on his or her back for at least 2-3 h.

\section{Results}

In all cases, donor loading onto the NSI spatula and donor insertion into the anterior chamber using the NSI were smooth and successful; no intraoperative complications were noted. There were no cases of graft dislocation or iatrogenic primary graft failure. Postoperative ECD was 2,187.2 cells $/ \mathrm{mm}^{2}$ indicating a mean cell loss of $14.8 \%$ compared to preoperative ECD $\left(2,568.0\right.$ cells $\left./ \mathrm{mm}^{2}\right)$. Mean best corrected visual acuity increased from 0.27 decimal to 0.8 at 6 months. Representative cases are shown below.

\section{Case 4}

An 83-year-old woman was referred to our hospital for decrease of visual acuity in her right eye. Prophylactic argon laser iridotomy had been performed in both of her eyes 5 years before. On slit-lamp biomicroscopy, she had bullous keratopathy in her right eye and visual 
acuity decreased to 0.15 (Fig. 2a). DSAEK using NSI was successfully performed and resulted in improved visual acuity of 0.7 with ECD of 2,487 cells $/ \mathrm{mm}^{2}$ (cell loss rate of $1.0 \%$ ) after 6 months (Fig. 2b).

\section{Case 5}

A 76-year-old woman was referred to our hospital for decrease of visual acuity in her right eye. Prophylactic argon laser iridotomy had been performed in both of her eyes 2 years before. Her right eye was aphakic due to previous complicated cataract surgery. On slit-lamp biomicroscopy, she had mild bullous keratopathy in her right eye and visual acuity was 0.9 (Fig. 2c). Simultaneous surgery of intraocular lens scleral fixation followed by DSAEK using NSI was successfully performed and resulted in improved visual acuity of 1.0 with ECD of 2,700 cells $/ \mathrm{mm}^{2}$ (ECD increased by $0.9 \%$ ) after 6 months (Fig. $2 \mathrm{~d}$ ).

\section{Discussion}

Currently, there are several donor inserters for DSAEK available in the United States; these include the TEG [7], NCI [8], and EndoSerter (CorneaGen ${ }^{\mathrm{TM}}$, Seattle, WA, USA). In one study that used the TEG for DSAEK, the mean endothelial cell loss rate after 12 months was reported to be as low as 16.3\% (100 eyes) [7]. We have previously tested the TEG in Japanese eyes and reported that the mean ECD cell loss rate was $22.9 \%$ at 6 months [12]. With the use of the NCI, the mean endothelial cell loss rate after 6 months was reported to be $33 \%$ (50 eyes) [13]. We have also tested the NCI in Japanese eyes and reported that the mean ECD cell loss rate was $22.1 \%$ after 6 months [8]. When the EndoSerter was evaluated for DSAEK, the mean endothelial cell loss rate after 6 months was reported to be $28.3 \%$ (46 eyes) compared to $44.1 \%$ (38 eyes) using the forceps technique [14]. Collectively, all of these inserters have been successfully used to prevent the initial severe endothelial cell loss after DSAEK. However, only the NCI has been commercially available in Japan; therefore, the use of the Busin glide has predominated in Japan.

Previously, Nakatani and Murakami [15] evaluated 3-year outcomes of DSAEK for patients with BK-ALI and reported that the median endothelial cell loss using the Busin glide pull-through technique was $20.3 \%$ at 6 months. In the present study, we evaluated the NSI for donor insertion during DSAEK for BK-ALI. This device utilizes BSS fluid flow to push the donor into the anterior chamber; this novel concept enables safe donor insertion and complete endothelial cell protection. During surgery, no cases experienced difficulties during donor loading into the NSI, and no intraoperative complications were noted. Results showed that postoperative ECD after 6 months was 2,187.2 cells $/ \mathrm{mm}^{2}$, indicating a mean cell loss rate of $14.8 \%$; this is comparable to or better than that achieved with other DSAEK insertion devices. However, it is somewhat difficult to compare these outcomes with those achieved in eyes of patients from other countries, since the causative diseases of bullous keratopathy in Asian countries are quite different from those of Western countries [11]. Previously, Soma and colleagues [9] reported that the NSI technique resulted in less endothelial cell damage compared with a pull-through technique in ex vivo experiments. Additionally, they reported that the endothelial cell loss rate after 6 months was as low as $18.2 \%$ when compared to that of the Busin glide technique (46.5\%) [10].

Some concerns may exist pertaining to the use of dispersive OVDs during NSI insertion. However, as evidenced by clinical studies, there have been no adverse effects from using dispersive OVDs for NSI. The OVD actually protects the endothelial cells while simultaneously 
enabling smooth donor push-in using BSS flow. However, the use of excessive OVD may result in unexpected complications such as interface migration of the OVD. Therefore, an appropriate amount of OVD is strongly recommended. Concerns may also exist pertaining to the risk of donor rotation in the anterior chamber after insertion, although, to date, we have not experienced this complication. However, meticulous care should be taken during donor insertion into the anterior chamber.

Our current study has some limitations in that it is a retrospective design, lacking controls, and involving only a small number of cases. An additional study using a larger number of patients will be required to fully validate the usefulness and potential advantages of this new donor inserter.

In conclusion, clinical outcomes for BK-ALI using the NSI during DSAEK were comparable or better than those achieved with conventional DSAEK insertion techniques in this small preliminary case series.

\section{Statement of Ethics}

This prospective non-comparative study was approved by the Ethical Committee of Kanazawa University Graduate School of Medical Science (approval number 2018-166 [2917]) and followed the tenets of the Declaration of Helsinki.

\section{Disclosure Statement}

No author has a financial or proprietary interest in any material or method mentioned in this article.

\section{Funding Sources}

A Grant-in-Aid for Scientific Research (C) KAKENHI, Japan (No. 19K09947).

\section{Author Contributions}

A.K. designed the study and wrote the initial draft of the manuscript. H.Y., T.N., N.M., and K.S. contributed to analysis and interpretation of data and assisted in the preparation of the manuscript. All other authors have contributed to data collection and interpretation and critically reviewed the manuscript. All authors approved the final version of the manuscript and agree to be accountable for all aspects of the work in ensuring that questions related to the accuracy or integrity of any part of the work are appropriately investigated and resolved.

\section{References}

1 Gorovoy MS. Descemet-stripping automated endothelial keratoplasty. Cornea. 2006 Sep;25(8):886-9.

2 Price MO, Gorovoy M, Benetz BA, Price FW Jr, Menegay HJ, Debanne SM, et al. Descemet's stripping automated endothelial keratoplasty outcomes compared with penetrating keratoplasty from the Cornea Donor Study. Ophthalmology. 2010 Mar;117(3):438-44. 
3 Terry MA, Saad HA, Shamie N, Chen ES, Phillips PM, Friend DJ, et al. Endothelial keratoplasty: the influence of insertion techniques and incision size on donor endothelial survival. Cornea. 2009 Jan;28(1):24-31.

4 Mehta JS, Por YM, Beuerman RW, Tan DT. Glide insertion technique for donor cornea lenticule during Descemet's stripping automated endothelial keratoplasty. J Cataract Refract Surg. 2007 Nov;33(11): 1846-50.

5 Busin M, Bhatt PR, Scorcia V. A modified technique for descemet membrane stripping automated endothelial keratoplasty to minimize endothelial cell loss. Arch Ophthalmol. 2008 Aug;126(8):1133-7.

6 Kobayashi A, Yokogawa H, Sugiyama K. Descemet stripping with automated endothelial keratoplasty for bullous keratopathies secondary to argon laser iridotomy - preliminary results and usefulness of doubleglide donor insertion technique. Cornea. 2008 Sep;27 Suppl 1:S62-9.

7 Khor WB, Mehta JS, Tan DT. Descemet stripping automated endothelial keratoplasty with a graft insertion device: surgical technique and early clinical results. Am J Ophthalmol. 2011 Feb;151(2):223-32.e2.

8 Kobayashi A, Yokogawa H, Sugiyama K. Clinical results of the Neusidl Corneal Inserter(®), a new donor inserter for Descemet's stripping automated endothelial keratoplasty, for small Asian eyes. Ophthalmic Surg Lasers Imaging. 2012 Jul;43(4):311-8.

9 Soma T, Koh S, Maeda N, Mitomo K, Quantock AJ, Nishida K. New Graft Insertion Device for Descemet Stripping Automated Endothelial Keratoplasty. Cornea. 2017 Nov;36(11):1432-6.

10 Soma T, Koh S, Oie Y, Maruyama K, Tsujikawa M, Kawasaki S, et al. Clinical evaluation of a newly developed graft inserter (NS Endo-Inserter) for Descemet stripping automated endothelial keratoplasty. Clin Ophthalmol. 2018 Dec;13:43-8.

11 Nishino T, Kobayashi A, Yokogawa H, Mori N, Masaki T, Sugiyama K. A 10-year review of underlying diseases for endothelial keratoplasty (DSAEK/DMEK) in a tertiary referral hospital in Japan. Clin Ophthalmol. 2018 Aug;12:1359-65.

12 Yokogawa H, Kobayashi A, Sugiyama K. Clinical evaluation of a new donor graft inserter for Descemet's stripping automated endothelial keratoplasty. Ophthalmic Surg Lasers Imaging. 2012 Jan-Feb;43(1):50-6.

13 Terry MA, Straiko MD, Goshe JM, Shamie N, Shah A, Alqudah AA, et al. Endothelial keratoplasty: prospective, randomized, masked clinical trial comparing an injector with forceps for tissue insertion. Am J Ophthalmol. 2013 Jul;156(1):61-68.e3.

14 Foster JB, Swan KR, Vasan RA, Greven MA, Walter KA. Small-incision Descemet stripping automated endothelial keratoplasty: a comparison of small-incision tissue injector and forceps techniques. Cornea. 2012 Jan;31(1):42-7.

15 Nakatani S, Murakami A. Three-year outcome of Descemet stripping automated endothelial keratoplasty for bullous keratopathy after argon laser iridotomy. Cornea. 2014 Aug;33(8):780-4.

The corresponding investigator (A.K.) has full access to all data in the study and takes responsibility for the integrity of the data and accuracy of the data analysis 


\section{Case Reports in Ophthalmology}

\begin{tabular}{l|l}
\hline Case Rep Ophthalmol 2019;10:357-364 \\
\hline DOI: 10.1159/000503441 & $\begin{array}{l}\text { ○ 2019 The Author(s). Published by S. Karger AG, Basel } \\
\text { www.karger.com/cop }\end{array}$ \\
\hline
\end{tabular}

Kobayashi et al: Clinical Evaluation of the NS Endo-Inserter, a Novel Donor Inserter for Descemet's Stripping Automated Endothelial Keratoplasty
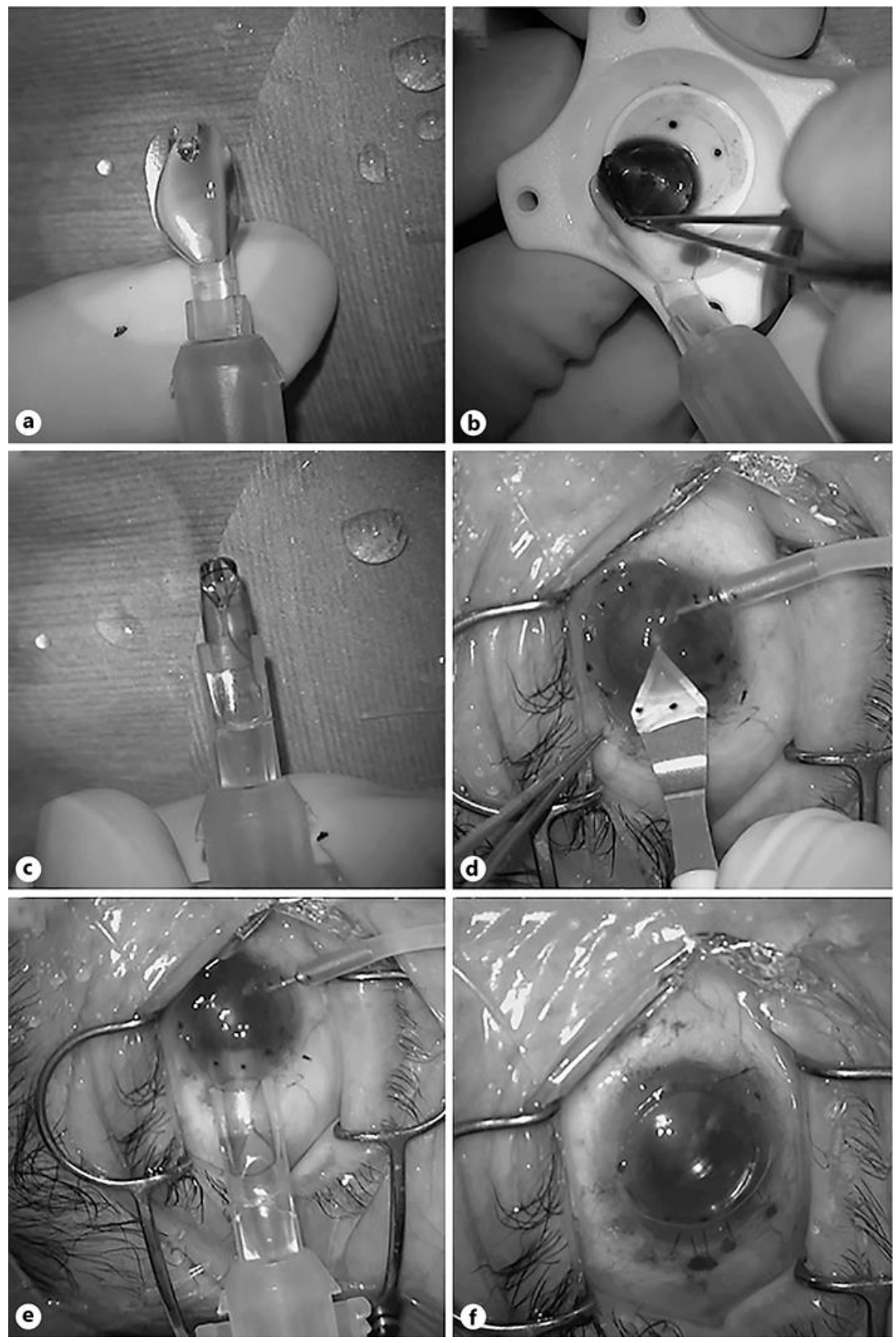


\section{Case Reports in Ophthalmology}

\begin{tabular}{l|l}
\hline Case Rep Ophthalmol 2019;10:357-364 \\
\hline DOI: 10.1159/000503441 & $\begin{array}{l}\text { @ 2019 The Author(s). Published by S. Karger AG, Basel } \\
\text { www.karger.com/cop }\end{array}$ \\
\hline
\end{tabular}

Kobayashi et al.: Clinical Evaluation of the NS Endo-Inserter, a Novel Donor Inserter for Descemet's Stripping Automated Endothelial Keratoplasty

Fig. 1. a-f DSAEK using the NS Endo-Inserter (NSI). a Before loading the donor onto the NSI, the syringe is filled with balanced salt solution. $\mathbf{b}$ The $8.0-\mathrm{mm}$ diameter donor endothelial lamella is then placed onto the platform with the endothelial cell surface facing upward. An appropriate ophthalmic viscoelastic device is applied to the endothelial surface of the graft. c The flexible platform with the graft is gently pulled into the cartridge resulting in coiling of the donor. $\mathbf{d}$ A 4.6-mm clear corneal incision using a special DSAEK knife is created. e The graft and balanced salt solution are then delivered into the anterior chamber by gentle depression of the syringe plunger through the clear corneal incision. Before insertion of the NSI, the anterior chamber maintainer was turned off. $f$ After securing the wound with an interrupted 10-0 nylon suture, air is injected into the anterior chamber to press the donor tissue against the recipient cornea. Corneal massage was performed in all cases to eliminate residual fluid at the recipient-donor interface.
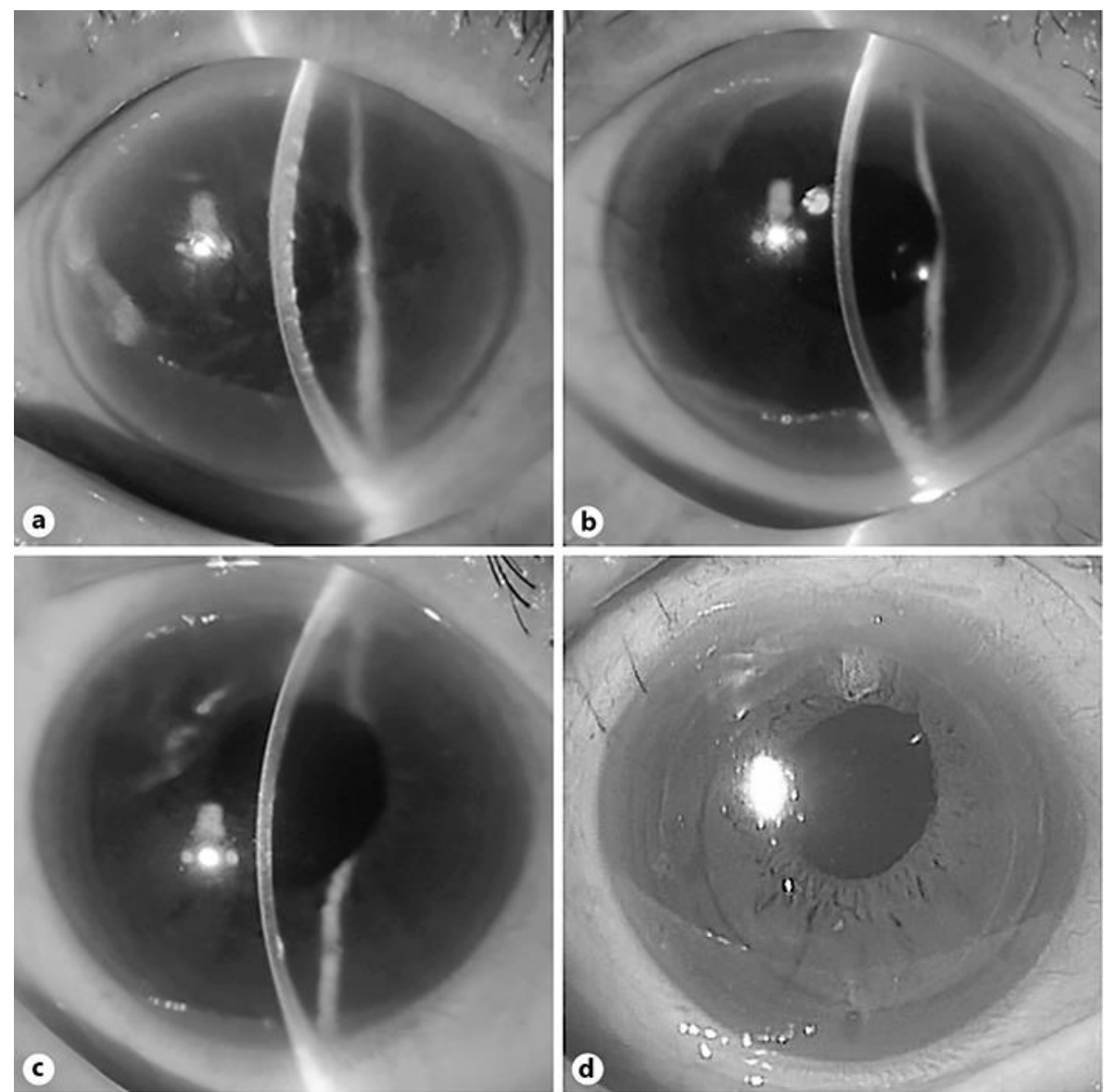

Fig. 2. Representative slit-lamp photo before and after DSAEK using the NS Endo-Inserter (NSI). a Slit-lamp photo of case 4 (83-year-old woman). Bullous keratopathy due to argon laser iridotomy was noted. b After 6 months of DSAEK using the NSI. Cornea was crystal clear and visual acuity improved to 0.7. c Slit-lamp photo of case 5 (76-year-old woman). Bullous keratopathy due to argon laser iridotomy with aphakia was noted. $\mathbf{d}$ After 6 months of DSAEK using the NSI and intraocular lens scleral fixation. Cornea was clear with intraocular lens in good position, and visual acuity improved to 1.0. 